DOI: $10.4274 /$ gulhane.galenos.2020.1426

Gulhane Med J 2021;63:313-315

\title{
Malignant phyllodes tumor in a young woman with multiple
} recurrences

\author{
(1) Zahra Aminparast ${ }^{1,2}$, (1) Masoud Sadeghi ${ }^{3}$, (1) Mazaher Ramezani ${ }^{4}$ \\ ${ }^{1}$ Kermanshah University of Medical Sciences, Students Research Committee, Kermanshah, Iran \\ ${ }^{2}$ Kermanshah University of Medical Sciences, Clinical Research Development Center, Imam Reza Hospital, Kermanshah, Iran \\ ${ }^{3}$ Kermanshah University of Medical Sciences, Medical Biology Research Center, Kermanshah, Iran \\ ${ }^{4}$ Kermanshah University of Medical Sciences, Imam Reza Hospital, Molecular Pathology Research Center, Kermanshah, Iran
}

Date submitted:

02.10.2020

Date accepted:

07.12.2020

Online publication date:

15.12.2021

\section{Corresponding Author:}

Mazaher Ramezani, M.D.,

Kermanshah University of Medical

Sciences, Imam Reza Hospital,

Molecular Pathology Research Center,

Kermanshah, Iran

mazaher_ramezani@yahoo.com

ORCID:

orcid.org/0000-0003-1897-7410

Keywords: Malignant phyllodes tumor, young woman, surgery, recurrence

\begin{abstract}
Phyllodes tumor (PT) is an uncommon tumor of primary breast origin, constituting about $1 \%$ of the breast tumors. Most of the PTs are benign, but some have malignant potential. A 22-year-old single female was referred with a history of left breast mass since she was 13 years old. The diagnosis was fibroadenoma and she had a history of 2 surgeries (three and six years ago). Left breast mass had recurred since last year. Ultrasound examination showed 3 masslike hypoechoic lesions without calcification. On core needle biopsy, the pathologist reported borderline PT. On mastectomy, the report changed to malignant PT with 10 mitoses/10 HPF. The greatest tumor diameter was measured as $10+8+6 \mathrm{~cm}$. The PT has unpredictable clinical behavior and has a high risk of recurrence regardless of histologic subtype. Pathologists and clinicians should be aware of this tumor type in younger individuals for a better diagnosis and treatment.
\end{abstract}

\section{Introduction}

"Fibroepithelial lesions" consist of fibroadenoma (FA) and phyllodes tumor (PT). FAs are more common in young females but PTs are often seen in the fourth and fifth decades of life. The $\mathrm{PT}$ is uncommon tumor of primary breast origin, constituting about $1 \%$ of the breast tumors $(1,2)$. Breast lesion is rare in adolescents and young population but FAs are the most common tumors at this age. Only $20 \%$ of PTs in adolescents and less than $10 \%$ of PTs before the age of 20 years are reported $(1,3,4)$. Large and rapidly growing mass in the breast is a key feature of PT (4). Most of the PTs are benign, but some have malignant potential and can behave like sarcoma with blood born metastasis to various organs (1). The World Health Organization has defined several histological criteria to differentiate FA from PT and for PT classification (5). Here we report a rare case of malignant PT in a young patient with multiple recurrences.

\section{Case Presentation}

A 22-year-old single female patient was referred on August $1^{\text {st }}, 2019$ with a history of left breast mass since she was 13 . The diagnosis was FA and she had a history of 2 surgeries ( 6 and 3 years ago). Left breast mass had recurred since last year. Drug 
and family histories were unremarkable and vital signs were stable. Physical examination was unremarkable except for left breast mass. The patient had the report of core needle biopsy on July $2^{\text {nd }}$, which showed a spindle cell tumor compatible with PT. Mitotic figures were about 4 mitoses/10 HPF and mild atypia without necrosis was present. The pathologist suggested low grade (borderline) PT on core needle biopsy (Figure 1) and recommended for complete excision and immunohistochemistry with CD34, CD31, CK, SMA, and Ki-67. Ultrasound examination showed 3 mass-like hypoechoic lesions without calcification and dimensions of $75 \times 41,75 \times 55$, and $66 \times 34 \mathrm{~mm}$ with lobulated margin. The radiologist commented on further evaluation due to large size and edema to rule out malignancy and PT. The patient underwent a total mastectomy on August $3^{\text {rd }}$. She experienced pruritus and urticaria after blood transfusion which were managed with hydrocortisone and diphenhydramine. The specimen that was sent for the pathologist consisted of breast tissue including skin measured $17 \times 15 \times 9 \mathrm{~cm}$ with 3 well-defined masses on cut surface measured 10,8 , and $6 \mathrm{~cm}$. The pathologist reported as

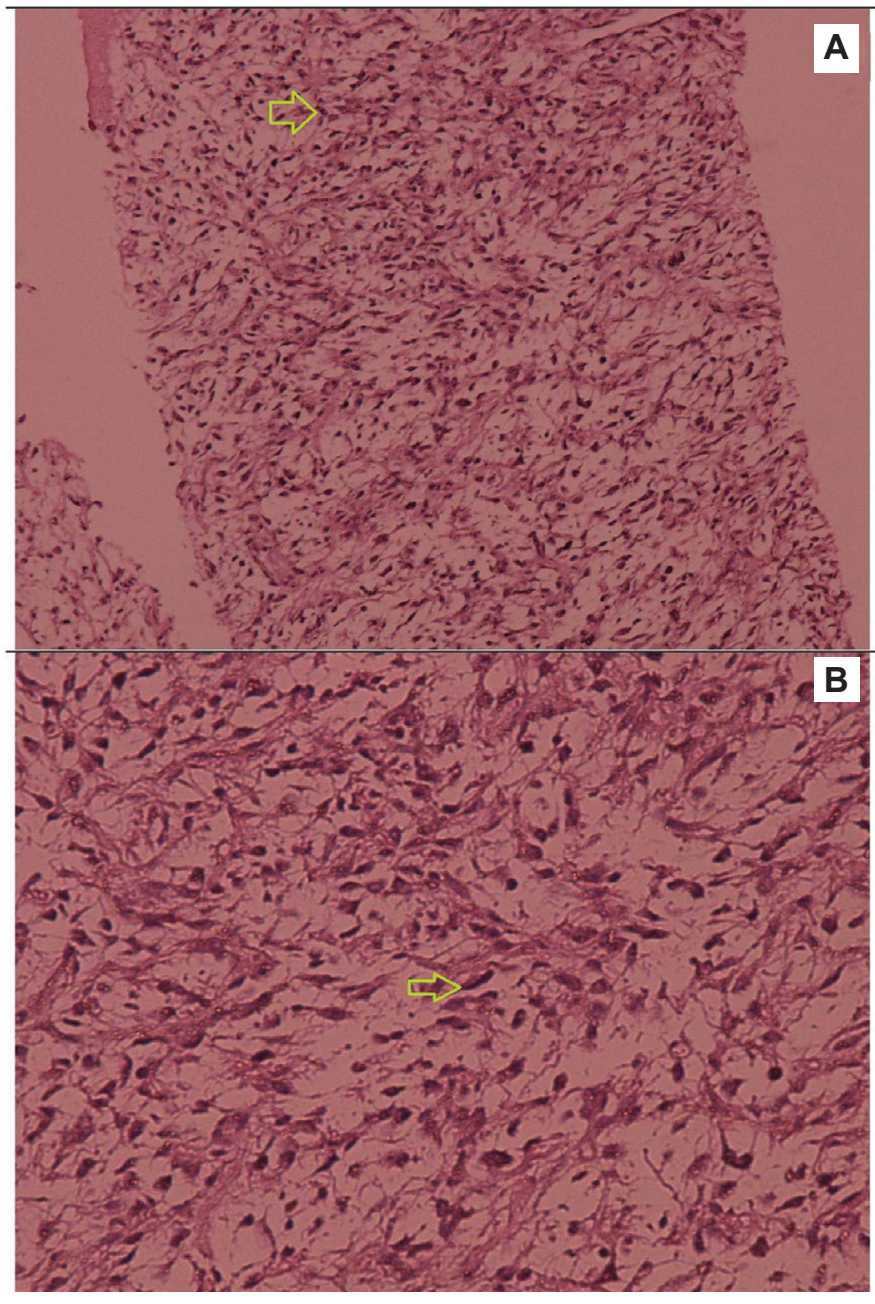

Figure 1. Borderline phyllodes tumor diagnosed on core needle biopsy. Tumor cells with spindle cell morphology (arrow). Hematoxylin-eosin stain: A) x100 and B) x200 magnifications compatible with malignant PT (Figure 2). Mitotic figures were 10 mitoses/10 HPF. The greatest tumor diameter was measured as $10+8+6 \mathrm{~cm}$ (24 cm, multiple). Inferior and medial margins were involved by tumor. On $26^{\text {th }}$ August, the patient was readmitted for the surgery of remnants of the tumor. Physical examination showed a surgical scar at the mastectomy site with a palpable $1 \mathrm{~cm}$ mass. The specimen consisted of one piece of breast tissue measured $17 \times 3 \times 2 \mathrm{~cm}$ including skin $(17 \times 0.3 \mathrm{~cm})$. The pathologist found no remnant of the tumor with unremarkable surgical margins. On $19^{\text {th }}$ May 2020, the computed tomography was done with markers for planning of radiation therapy. Written informed consent was obtained from the patient for the report.

\section{Discussion}

The differential diagnosis of breast lesion in young and adolescent population includes giant FA, juvenile FA and PT. Clinical features of PT are the same as FA and include welldefined, movable and painless lesion. The only difference is more rapid growth in PTs $(2,3)$. PT's size has been reported from 1 to $40 \mathrm{~cm}$; about $20 \%$ of them are larger than $10 \mathrm{~cm} \mathrm{(6).}$ Etiologic causes and risk factors of PTs are unknown but more reported in Asian races (1).

Diagnosis of PT is based on clinical symptoms, radiological findings and histological examination (6). Preoperative diagnosis is important but there is no standard protocol for diagnosis $(6,7)$. Ultrasonography and mammography cannot differentiate PT from other benign breast tumors (1). The use of cytologic examination in the diagnosis of PT has been controversial but core needle biopsy has sensitivity of about $70 \%(1,7,8)$. $\mathrm{PT}$ in core needle biopsy may be mistaken for low grade myofibromatosis, solitary fibrous tumor, FA and leiomyosarcoma (9). PT is often benign (35-64\%), while malignant PT is larger than borderline and benign PTs and it accounts for $6.5-27 \%$

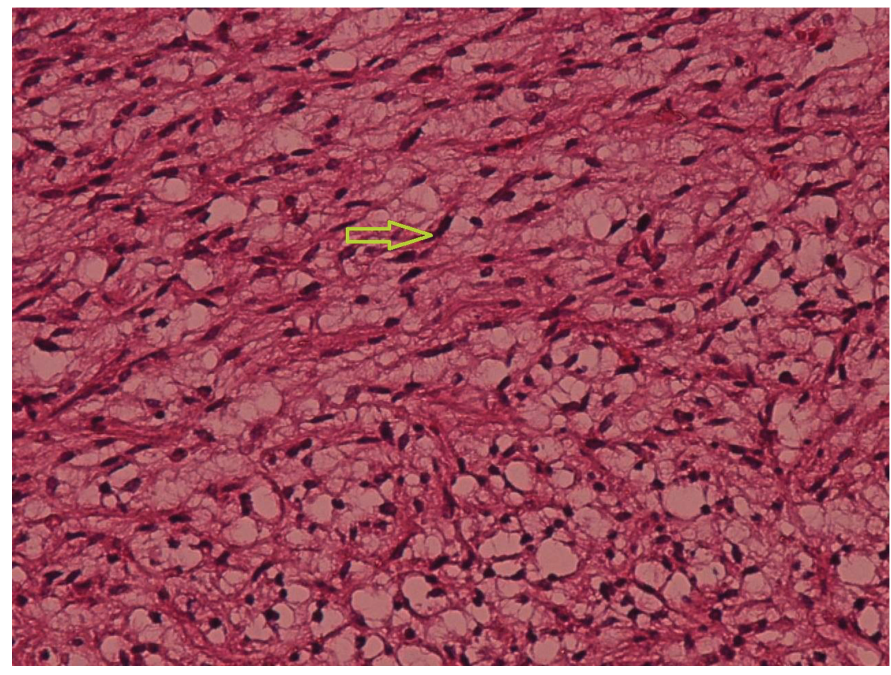

Figure 2. Malignant phyllodes tumor diagnosed on mastectomy specimen. Tumor cells with spindle cell morphology (arrow). Hematoxylin-eosin stain: x200 magnification 
of cases $(1,4)$. It is difficult to differentiate between malignant PT and pure sarcoma. In breast, rhabdomyosarcoma and liposarcoma are more common than fibrosarcoma (1). A clonal analysis showed monoclonal stromal cells and polyclonal epithelial cells. The study of the profile can help the evaluation of malignant transformation (6).

Thirty percent of malignant PTs are due to FA transformation and cases of transformation from benign into malignant $P$ Ts have been reported $(4,6,10)$. Decreased expression of beta catenin and ER with increased expression of EGFR, P53, C-kit, CD34, and Ki-67 are associated with malignant PTs. Among these markers, P53 expression is the most accepted one (6). MED12 (Mediator of RNA polymerase 2 transcription) expression is associated with long time survival and its absence is related to high recurrence rate of PTs $(5,10)$. The relationship between malignant PTs and a further recurrence of the tumor is unknown. About $20 \%$ of malignant PTs are associated with distant metastasis and more metastases are without local recurrence of the PT (8). A research has reported malignant PTs with six episodes of local relapse without evidence of distant metastasis (11). One study has reported that stromal cells pleomorphism and histologic grade are associated with local recurrence rate and distant metastasis respectively (8). Malignant PT is more common than benign PT in adolescent population. Adolescent and young females have more recurrence rate compared to adult population (4). Free margins and adjuvant radiotherapy are the best treatment to control local recurrence in borderline and malignant PT and patients need to be closely followed up with breast examination and ultrasonography at least for 30 months because average recurrence time is less than 24 months (8). Multiple PTs are rare (7). This study reported three separated malignant PTs.

\section{Conclusion}

PT is a mysterious tumor with unpredictable clinical behavior and has a high risk of recurrence regardless of histologic subtype. Pathologist and clinician must be aware of this tumor in young patients for better diagnosis and treatment.

\section{Acknowledgment}

The authors would like to thank the Clinical Research Development Center of Imam Reza Hospital for Consulting Services.

\section{Ethics}

Informed Consent: Written consent was obtained from the patient for reporting the case report.
Peer-review: Externally peer-reviewed.

\section{Authorship Contributions}

Concept: M.R., Design: Z.A., Data Collection or Processing: M.R., Analysis or Interpretation: Z.A., M.S., Literature Search: M.S., M.R., Writing: M.R.

Conflict of Interest: No conflict of interest was declared by the authors.

Financial Disclosure: This study supported by Kermanshah University of Medical Sciences, Kermanshah, Iran (project code: 3010733).

\section{References}

1. Albalawi IA. A huge phyllodes tumor in the breast: a case report. Electron Physician. 2018;10:6951-6955.

2. Lee JY. Giant phyllodes tumor of the breast with diffuse myxoid changes in an adolescent girl: a case report. J Surg Case Rep. 2017;2017:rjx019.

3. Makhija D, Shah H, Bothra J, Jayaswal S. An adolescent with a phyllodes tumor: A case report and review. Int $J$ Pediatr Adolesc Med. 2016;3:180-183.

4. Makar GS, Makar M, Ghobrial J, Bush K, Gruner RA, Holdbrook T. Malignant Phyllodes Tumor in an Adolescent Female: A Rare Case Report and Review of the Literature. Case Rep Oncol Med. 2020;2020:1989452.

5. Câmara S, Gonzàlez-Farré X, Vargas-Moniz J. Giant phyllodes tumour - Case report, oncoplastic treatment and literature controversies. Revista de Senología y Patología Mamaria. 2017;30:79-84.

6. Pornchai S, Chirappapha P, Pipatsakulroj W, et al. Malignant transformation of phyllodes tumor: a case report and review of literature. Clin Case Rep. 2018;6:678-685.

7. Bhasin SK, Kumari S, Kumar V, Saini P, Sharma G, Akram M. Bilateral benign giant phyllodes tumor in an adolescent female: a rare case report. Int Surg J. 2014;1:177-180.

8. Sawalhi S, Al-Shatti M. Phyllodes tumor of the breast: a retrospective study of the impact of histopathological factors in local recurrence and distant metastasis. Ann Saudi Med. 2013;33:162-168.

9. Takenaka M, Toh U, Otsuka $\mathrm{H}$, et al. Giant malignant phyllodes tumor: a case report. Kurume Med J. 2011;58:6772.

10. Wang Q, Su J, Lei Y. Recurrent malignant phyllodes tumor of the breast: A case report. Medicine (Baltimore). 2017;96:e9069.

11. limori N, Kashiwagi S, Ishikawa T, et al. Mammary phyllodes tumor with six episodes of a relapse: a case report. J Med Case Rep. 2017;11:261. 